\title{
Mdm2 and Egfr Expression and Prognosis in 16 Pulmonary Artery Sarcomas
}

\author{
Li L ${ }^{1}$, Duan $\mathbf{X}^{1}$, Yang Sun $\mathbf{M}^{1}$, Wang $\mathrm{H}^{1}$, Song $\mathbf{Y}^{2}$, Lu $\mathbf{Y}^{1}$, Xu $\mathrm{H}^{1}$, Wang $\mathbf{Q}^{1}$, You $\mathbf{Y}^{1}$, \\ Buja $\mathrm{LM}^{3}$ and Zhao $\mathrm{B}^{3 *}$ \\ ${ }^{1}$ Department of pathology, Fuwai Hospital, Chinese Academy of Medical Sciences, China \\ ${ }^{2}$ Center for adult surgery, Fuwai Hospital, Chinese Academy of Medical Sciences, China \\ ${ }^{3}$ Department of Pathology and Laboratory medicine, McGovern Medical School, The University \\ of Texas, Houston
}

Research Article

Volume 5 Issue 1

Received Date: October 22, 2020

Published Date: February 11, 2021

DOI: $10.23880 /$ cprj-16000129

*Corresponding author: Bihong Zha, Department of Pathology and Laboratory medicine, McGovern Medical school, The University of Texas, 6431 Fannin Street MSB 2.274 Houston TX 77030, USA, Tel: 713-500-5316; Email: bihong.zhao@uth.tmc.edu

\section{Abstract}

There are few studies investigating the biomarker signatures and their relations with prognosis of pulmonary artery sarcoma patients. In the present study, we performed a cohort study on 16 cases of pulmonary artery sarcoma in an attempt to identify the potential diagnostic markers and molecular therapeutic targets of that vascular sarcoma. Patients who had undergone pulmonary artery sarcoma tumorectomy over $7 \frac{1}{2}$ years in our hospital were enrolled in this retrospective study. The resected tumors were analyzed by histopathology, the expression and mutation of Murine Double Minute 2 (MDM2), Epidermal Growth Factor Receptor (EGFR), and Platelet-Derived Growth Factor Alpha (PDGFRA) by immunohistochemistry (IHC) and fluorescence in situ hybridization (FISH). The correlations between patients' outcomes and tumor histological, IHC and FISH features were statistically analyzed. MDM2 immunostaining was seen mainly in intimal sarcoma. At univariate analysis, MDM2 expression had a positive correlation with shorter progression free survival (PFS). MDM2 and EGFR co-expression also had a positive correlation with shorter PFS and tumor metastasis. This study investigated MDM2, EGFR, and PDGFRA as potential biomarkers for diagnosis of pulmonary artery sarcoma and therapeutic targets.

Keywords: Pulmonary artery sarcoma; Clinicopathologic features; MDM2; EGFR; Prognostic factor

Abbreviations: MDM2: Murine Double Minute 2; EGFR: Epidermal Growth Factor Receptor; PDGFRA: PlateletDerived Growth Factor Alpha; IHC: Immunohistochemistry; PFS: Progression free survival; CGH: Comparative genomic hybridization; OS: Overall survival; PBS: Phosphate-buffered saline.

\section{Introduction}

Primary sarcomas of the pulmonary artery are uncommon and remain a subject of case reports [1,2]. Most pulmonary artery sarcomas are thought to arise from pluripotent intimal cells, and the term intimal sarcoma (IS) has been proposed [3,4]. By WHO classification of tumor, IS are malignant mesenchymal tumors arising in large arterial blood vessels of the systemic and pulmonary circulation. Intima sarcomas typically are poorly differentiated malignant mesenchymal tumors with fibroblastic or myofibroblastic differentiation, which show intraluminal growth pattern with obstruction of the lumen of the vessels of origin and the seeding of emboli to peripheral organs. Other types of luminal sarcomas include rhabdomyosarcoma, osteogenic sarcoma and angiosarcoma, which have more distinct histologic features and immunohistochemical profiling [4].

Up to now, there have been few studies about the biological behavior and prognosis of IS. In Tavora and his colleagues' study [5]. They found that histologic diversity of pulmonary artery sarcomas had association with prognosis. 


\section{Clinical Pathology \& Research Journal}

Intimal sarcoma with myofibroblastic differentiation has a better prognosis. To distinguish intimal sarcoma as a different entity from other sarcomas will help cardiac surgeons and oncologists to decide the matter of surgery and chemotherapy.

In recent years, by comparative genomic hybridization $(\mathrm{CGH})$, recurrent amplification of the regions $4 \mathrm{q} 12$ and 12q13-q15 was described in a subset of IS [6,7]. Amplification of the chromosomal region 12q13-q15 was reported in $75 \%$ of these tumors [6,7]. Multiple genes in this region, such as MDM2, SAS, CDK4, and GLI1, are frequently amplified in many types of human cancer $[7,8]$. In a more recent study, deregulated copy numbers of PDGFRA/PDGFRB, KIT, and EGFR were described in pulmonary IS cases [9].

Till now the prognostic significance of MDM2, PDGFRA and EGFR expression together with various morphological features (proliferation, necrosis and tumor grade) was not investigated in pulmonary artery sarcoma. The aim of this study was also to investigate the relationship between expression levels of MDM2, PDGFRA and EGFR and patient prognosis. And, the prognostic significance of tumor morphologic features and surgery pattern was also investigated in pulmonary sarcoma.

\section{Materials and Methods}

\section{Patients}

Between January 2011 and June 2018, sixteen patients with primary pulmonary artery sarcoma were identified. The patients' clinical data including age, sex, signs/symptoms and survival time after surgery were collected and reviewed. The end of follow-up was November, 2018. All cases were confirmed to be of pulmonary primary site based on clinical chart review, lacking of history of sarcoma at a distant site, and radiographic, intraoperative, and/or gross pathologic confirmation. IS and other sarcomas were diagnosed by two separate pathologists according to WHO classification and FNCLCC grading system $[10,11]$. The expression of MDM2 protein and gene amplification were performed as auxiliary tests. Surgery patterns included radical resection, complete tumorectomy, and partial tumorectomy. Radical resection means that surrounding structures such as the right ventricular outflow, main pulmonary artery trunk, and lung were resected. Complete resection means tumor was thoroughly resected with clear surgical margins or pulmonary endarterectomy. Partial resection means only partial tumor was rejected because of invasion of tumor into pulmonary artery media and/or adventitia or right ventricle.

Progression free survival (PFS) was assessed from the date of enrolment to the date of disease progression to the first-line or to the date of death, whichever occurred first. Overall survival (OS) was assessed from the date of enrolment to the date of death. This study complied with the Declaration of Helsinki and was approved by the Institutional Review Board of Fuwai Hospital and the patients' consent was obtained.

\section{Histopathological and Immunohistochemistry Examination}

Histopathologic examination was performed on formalin-fixed, paraffin-embedded tumor tissues. Five micrometer sections were used for haematoxylin and eosin (H\&E) preparations.

The presence of necrosis, mitotic rate and tumor differentiation were evaluated on H\&E stained sections and quantified using a score system $[10,11]$. Tumor necrosis score was 0 : absent; $1:<50 \% ; 2:>50 \%$ of the whole tumor section examined [12]. Mtiotic activity score was 1:0-9 mitoses/10 HPF; 2:10-19 mitoses/10 HPF; 3: $\geq 20$ mitoses/10 HPF. Tumor differentiation score was accordance with FNCLCC system for sarcoma grading system. For example, intimal sarcoma, exoskeletal osteosarcoma and dedifferentiated chondrosarcoma were 3 and well-differentiation chondrosarcoma was 1 in tumor differentiation. Combining tumor differentiation (scored 1 to 3), mitotic count (scored 1 to 3), and tumor necrosis (scored 0 to 2) was the final sarcoma grade. Grade 1 sarcoma had a score 2-3, grade 2 with a score 4-5, and grade 3 with a score 6-8.

Immunohistochemical staining was performed on formalin-fixed, paraffin-embedded tumor tissues as described in manufacturer's protocol; using antibodies against the following monoclonal (mc) and polyclonal (pc) antibodies: $\alpha$-smooth muscle actin ( $\alpha$-SMA; mc, $1 / 100$; DAKO), desmin (mc, 1/20; ICN), CD31 (mc, 1/50; DAKO), CD34 (mc, 1/10; DAKO), MDM2 (mc, 1/100; Invitrogen, Life Technologies), CD117 (pc, 1/250; DAKO), Ki67 (Clone MIB-1, Immunotech), EGFR (EGFR PharmDx Kit, DAKO), and PDGFRA (pc, 1/250; DAKO). Briefly, sections were deparaffinized and rehydrated. Antigen was recovered in $10 \mathrm{mmol} / \mathrm{L}$ sodium citrate buffer (pH 6.0). Endogenous peroxidase activity was inhibited using $3 \%$ hydrogen peroxide in methanol for 15 minutes. The sections were then blocked with $10 \%$ normal goat serum in phosphate-buffered saline (PBS). Sections were incubated with $100 \mu \mathrm{l}$ diluted primary antibodies overnight at $4^{\circ} \mathrm{C}$, followed by incubation with the IHC Detection Reagent (HRP, Rabbit. Cell Signaling Technology, Inc, CA) for 30 minutes at room temperature. The sections were then developed with a diaminobenzidine kit (DAB kit; Thermo Fisher Scientific Inc. MA, USA), and counter-stained with weak hematoxylin. The membranous expression of EGFR protein and the cytoplasmic expression of PDGFRA protein were reported using a three- 
tier system ranging from $1+$ (weak intensity) to $3+$ (strong intensity) [12,13]. Ki67 and MDM2 nuclear expression were counted by using Image-Pro plus 6.0 (for Windows) and expressed as percentage of positive cells over total tumor cells [12]. Ki67 percentage over 50\% was considered high. MDM2 percentage over 20\% was considered high expression [14].

\section{Fluorescence in Situ Hybridization}

FISH for MDM2 was performed using a commercially available Vysis LSI MDM2 Dual Color Probe (Abbott Molecular, Des Plaines, IL). FISH analyses were performed on 4- $\mu \mathrm{m}$ sections according to an established laboratory protocol, as previously described [15]. Slides were deparaffinized for $3 \times 10$ minutes in xylene, washed in $100 \%$ ethanol, air-dried, incubated in $2 \times$ sodium saline citrate (SSC) at $72^{\circ} \mathrm{C}$ for 40 minutes, incubated in a proteinase $\mathrm{K}$ solution $(500 \mathrm{mg} / \mathrm{mL}$ in $2 \times$ SSC; Roche, Meylan, France) at $45^{\circ} \mathrm{C}$ for 5 to 80 minutes, washed in $2 \times \mathrm{SSC}$ for $2 \times 3$ minutes at room temperature, and stored in $70 \%$ ethanol at $4^{\circ} \mathrm{C}$. The Vysis LSI MDM2 Spectrum Orange Probe (Abbott Molecular) combined with Vysis CEP 12 (D12Z3) Spectrum Green Probe (Abbott Molecular) was used. FISH images were captured using the Metafer Slide Scanning Platform (MetaSystems, Altlussheim, Germany). Each FISH slide was evaluated by 2 independent investigators in a blind manner. A minimum of 100 nuclei were counted on each slide. MDM2 amplification was defined as MDM2/CEP12 ratio $\geq 2.0$, while a ratio $<2.0$ were considered nonamplified. A ratio $<2.0$ with $>2$ signals was considered polysomic for CEP [12].

\section{Statistical Analysis}

All statistical tests were performed using SPSS software (version 17.0). Mean was calculated for quantitative variables included patient's age, scores of MDM2 andKi67, months of PFS and OS. Frequencies and percentages were calculated for qualitative variables like gender, PDGFRA and EGFR expression. Univariate (log rank Mantel Cox test) and multivariate (Cox Regression Proportional Hazards Model) analyses were performed to show any possible impact of molecular markers, pathological parameters and clinical features on PFS and OS.

\section{Results}

\section{Clinicopathological Findings}

The patients included 9 males and 7 females aged 25 to 78 years (mean, 47 years) at the time of diagnosis. The most common symptoms were of pulmonary embolism related, including chest pain, short of breath and nonproductive cough. Two patients already had metastasis to lung or bone at the time of diagnosis.

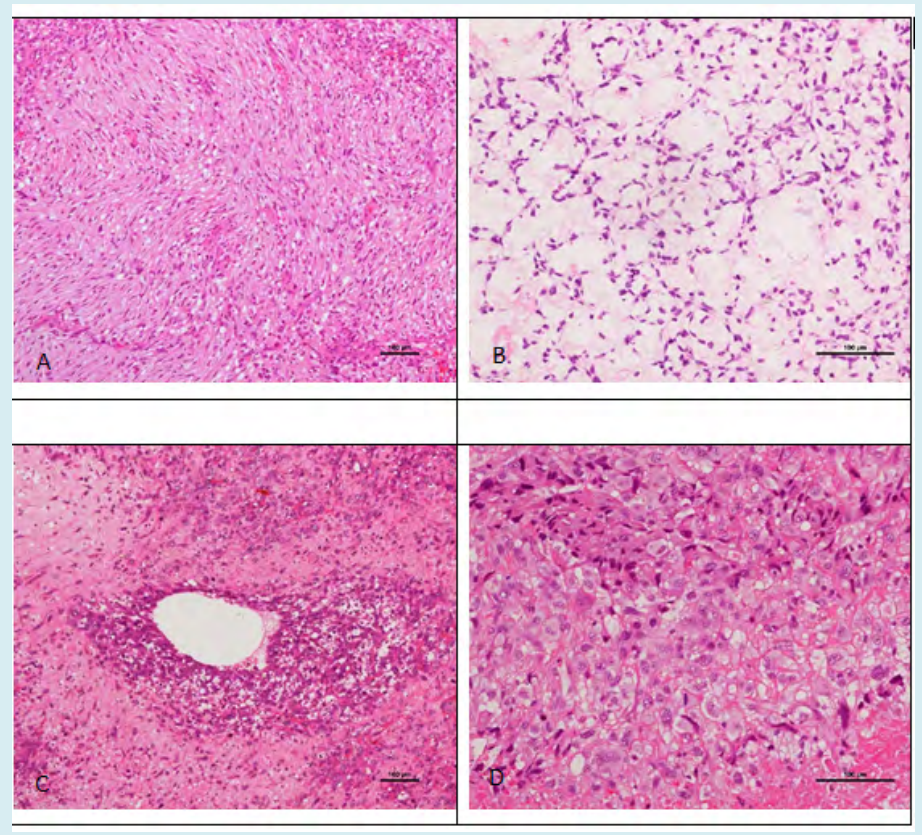

Figure 1: Histological feature of pulmonary artery intimal sarcoma. (A) Atypical spindle cells are arranged into the storiform pattern. (B) Mildly atypical spindle cells are embedded in the large myxoid areas, (C) The tumor cells show perivascular growth pattern. (D) The tumor cells demonstrated epitheloid appearance with conspicuous cytoplasm and enlarged nuclei. Hematoxylin-eosin staining, original magnification A, C × 100 and B, D $\times 200$. 


\section{Clinical Pathology \& Research Journal}

Histopathologically, the most common tumor was IS $(n=13)$; and other types included one osteosarcoma and two chondrosarcomas (Figure 1). Grossly, the pulmonary artery sarcomas were polypoid or multinodular masses attached to the inner vascular wall, and extended distally along the branches of the pulmonary artery. In two cases, the tumors invaded the pulmonary valve and/or right ventricular outflow. On histology, IS consists of mildly to severely atypical spindle cells with varying degrees of nuclear pleomorphism, mitosis and necrosis. Six ISs showed large myxoid areas (Figure 1B). 10 cases had epithelioid tumor cells and 5 cases showed perivascular growth pattern (Figure 1C).

Pulmonary artery osteosarcoma showed cytologically malignant spindle tumor cells associated with lace-like tumor bone (Figure 2).
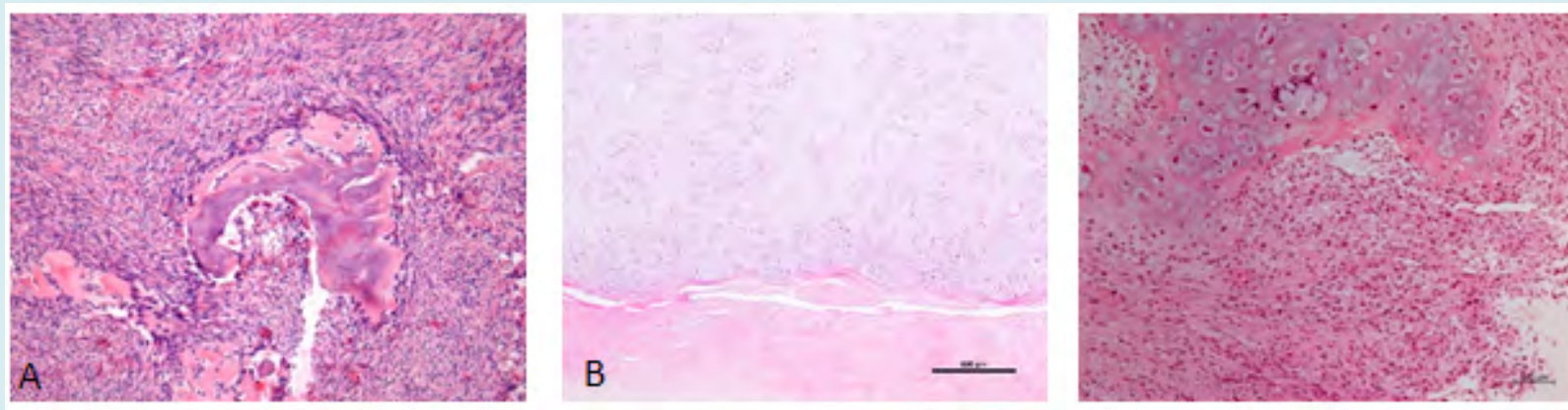

Figure 2: Histological feature of pulmonary osteosarcoma and chondrosarcoma (a) Osteosarcoma: malignant spindle tumor cell associated with lace-like tumor bone. (b) Grade I chondrosarcoma/atypical chondroma: abundant blue-grey matrix and irregularly shaped lobular cartilage varying in shape. The chondrocytes were mild atypical and binucleation tumor cells were frequently seen. The tumor did not invade underlaid internal elastic lamina of pulmonary artery (Arrow showed). (C) Dedifferentiated chondrosarcoma: there was an abrupt transition between the grade I chondrosarcoma and high-grade undifferentiated sarcoma. Hematoxylin-eosin staining, an original magnification $\times 200$, C x100. Elastic staining B $\times 40$.

Of two pulmonary artery chondrosarcomas, one was grade I chondrosarcoma/atypical chondroma and the other one was dedifferentiated chondrosarcoma. Grade I chondrosarcoma was characterized by abundant blue-grey matrix and irregularly shaped lobular cartilage varying in shape. The chondrocytes were mild atypical and binucleation tumor cells were frequently seen. Mitoses were rarely seen. In dedifferentiated chondrosarcoma, there was an abrupt transition between the grade I chondrosarcoma and highgrade undifferentiated sarcoma (Figure 2).

According to FNCLCC grading system, nine sarcomas were grade 3 ; four were grade 2 ; and three were grade 1 . The clinical, pathological and molecular features of sarcomas were detailed in Table 1.

\begin{tabular}{|c|c|c|c|c|c|c|c|c|c|c|c|c|}
\hline \multirow{2}{*}{ Case } & \multirow{2}{*}{ Sex } & \multirow{2}{*}{ Age } & \multirow{2}{*}{ Site } & \multirow{2}{*}{ Histologic type } & \multicolumn{4}{|c|}{ Immunostaining } & \multirow{2}{*}{$\begin{array}{c}\text { MDM2 } \\
\text { FISH }\end{array}$} & \multirow{2}{*}{ Surgical patten } & \multirow{2}{*}{ PFS(mths) } & \multirow{2}{*}{ oS(mths) } \\
\hline & & & & & MDM2 & PDGRA & EGFR & Ki67 & & & & \\
\hline 1 & M & 45 & PA & IS & $36.07 \%$ & 2 & 2 & 7.53 & + & Radical & 4 & 4 \\
\hline 2 & $\mathrm{~F}$ & 39 & $\mathrm{PA}$ & IS & $19.43 \%$ & 2 & 2 & 7.51 & - & Complete & 4 & 4 \\
\hline 3 & $\mathrm{~F}$ & 41 & $\mathrm{PA}$ & IS & $67.91 \%$ & 1 & 2 & 13.17 & + & Radical & 6 & 36 \\
\hline 4 & $\mathrm{~F}$ & 55 & $\mathrm{PA}$ & IS & $46.56 \%$ & 2 & 2 & 9.64 & + & Radical & 0 & 0 \\
\hline 5 & $\mathrm{M}$ & 46 & $\mathrm{PA}$ & IS & $36.89 \%$ & 0 & 0 & 5.49 & + & ND & 2 & 2 \\
\hline 6 & M & 62 & $\mathrm{PA}$ & IS & $47.95 \%$ & 0 & 2 & 20.01 & + & Radical & 3 & 9 \\
\hline 7 & M & 32 & $\mathrm{PA}$ & IS & $62.21 \%$ & 0 & 2 & 10 & + & Complete & 1 & 18 \\
\hline 8 & $\mathrm{M}$ & 41 & $\mathrm{PA}$ & IS & $75.06 \%$ & 0 & 1 & 35 & + & Partial & 6 & 6 \\
\hline 9 & M & 41 & PA & IS & $14 \%$ & 0 & 2 & 33.45 & - & ND & 1 & 1 \\
\hline 10 & $\mathrm{~F}$ & 46 & PA & Osteosarcoma & 0 & 0 & 0 & 3 & - & Radial & 24 & 24 \\
\hline
\end{tabular}




\section{Clinical Pathology \& Research Journal}

\begin{tabular}{|c|c|c|c|c|c|c|c|c|c|c|c|c|}
\hline $\mathbf{1 1}$ & M & 25 & PA & IS & 0 & 2 & 0 & 4.5 & - & Partial & 24 & 24 \\
\hline $\mathbf{1 2}$ & F & 53 & PA & IS & 0 & 0 & 0 & 9.76 & - & Partial & 3 & 5 \\
\hline $\mathbf{1 3}$ & F & 45 & PA & IS & $0-$ & 0 & 2 & 23.27 & - & Complete & 36 & 36 \\
\hline $\mathbf{1 4}$ & M & 48 & PA & IS & $15.30 \%$ & 0 & 0 & 18.58 & - & Complete & 6 & 6 \\
\hline $\mathbf{1 5}$ & M & 78 & PA & Chondrosarcoma & 0 & 0 & 0 & 1 & - & Radial & 22 & 22 \\
\hline $\mathbf{1 6}$ & F & 55 & PA & Chondrosarcoma & 0 & 0 & 0 & 15.4 & - & Complete & 5 & 5 \\
\hline
\end{tabular}

Table 1: Clinical and pathologic features of 16 cases of pulmonary artery sarcoma.

\section{Immunohistochemistry and Molecular Features}

To study the correlation between expression of MDM2, EGFR, and PDGFRA and tumor prognosis, we performed expression profiling of these biomarkers in the 16 enrolled subjects by
IHC and FISH (technical details are described in Materials and Methods section). By IHC, in 13 cases of IS, MDM2 positive expression was found in 9 (9/13); EGFR positive expression in $8(8 / 13)$ and PDGFRA positive in 5 (5/13) (Figure 3).

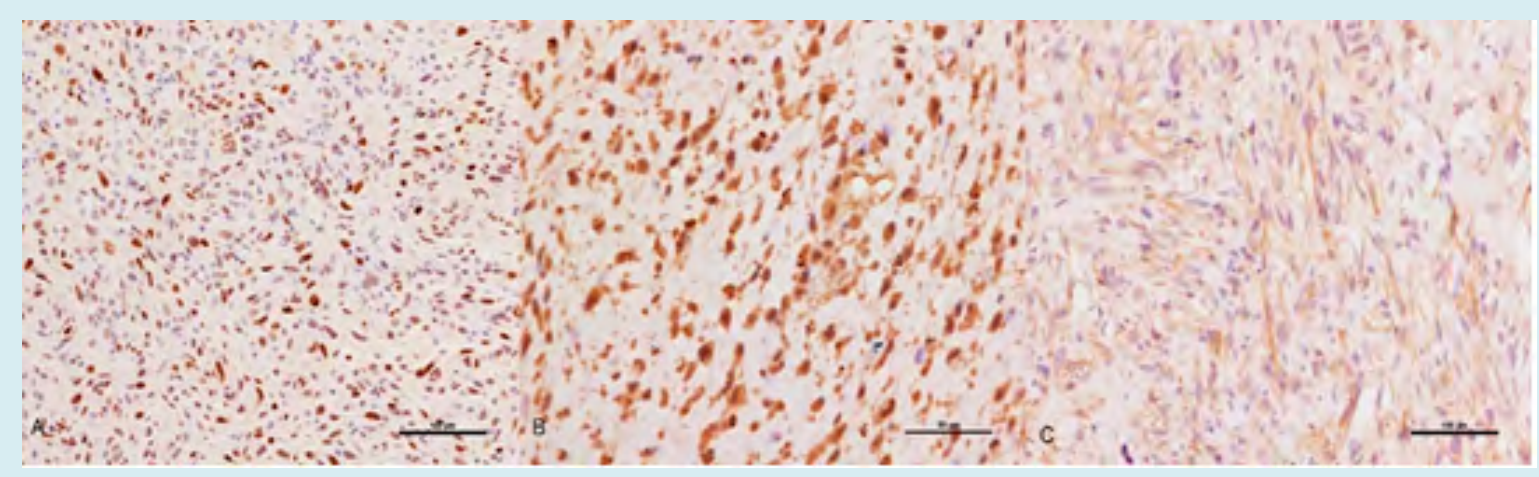

Figure 3: Immunohistochemical and FISH of MDM2 in pulmonary artery IS (A) Positivity expression of MDM2 in cell nuclei; (B) the presence of a large copy number of MDM2 gene organized in small clusters. Original magnification $(A \times 200, B \times 400)$.

Other three non-IS were all negative for MDM2, EGFR and PDGFRA. By FISH, four of 13 ISs $(30.8 \%, 4 / 13)$ coexpressed MDM2, PDGFRA and EGFR; whereas, none of three other sarcomas showed this coexpression. The grade of EGFR expression demonstrated positive correlation with MDM2 expression percentage (correlation coefficient: 0.548 , $\mathrm{p}=0.028)$. MDM2 FISH was performed in all 16 cases and MDM2 amplification was detected in 7 cases of IS (Figure 4).

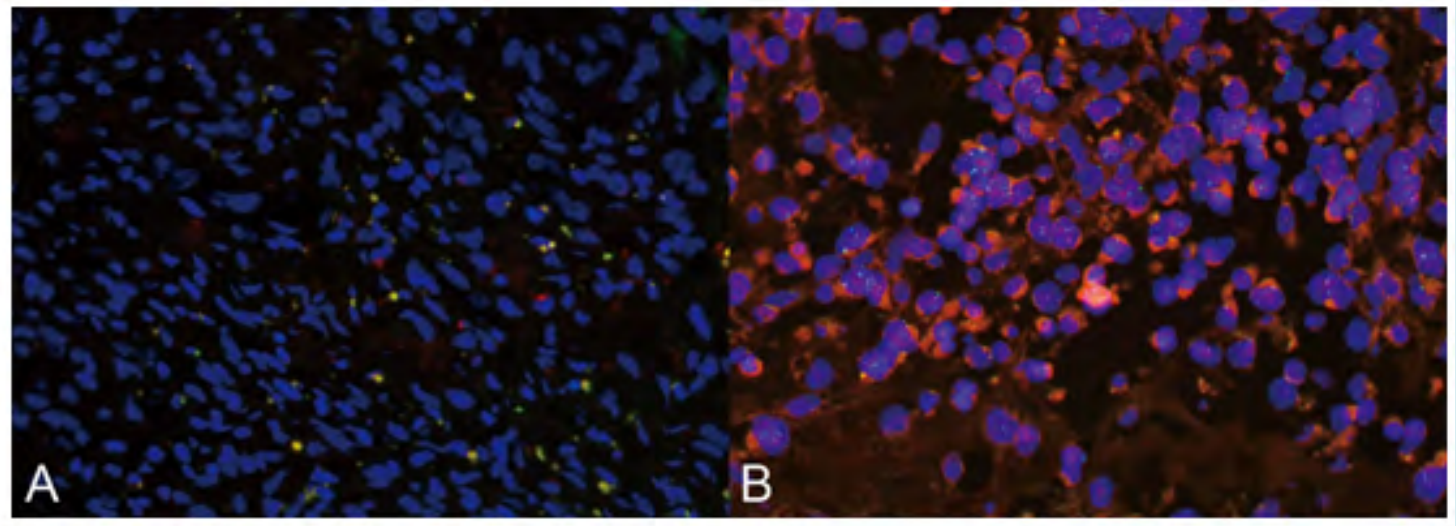

Figure 4: FISH analysis in pulmonary artery IS: the presence of a large copy number of MDM2 gene organized in small clusters (Original magnification $\times 400$ ). 


\section{Clinical Pathology \& Research Journal}

\section{Proliferation Index, Necrosis and Tumor Grade}

To investigate relationship between tumor grade and Ki67 index, we measured Ki67 index by IHC and compared with tumor grade. Intimal sarcoma had higher tumor grade compared to non-IS tumors $(2.62 \pm 0.65$ vs $1.33 \pm 0.68$, $\mathrm{p}=0.007)$. There was not difference of ki67 index and necrosis in between IS and non-IS (15.15 \pm 10.23 vs $6.47 \pm 7.80$, $p=0.193$ and $1.15 \pm 0.90$ vs $0.67 \pm 1.16, p=0.432$ ). These results indicate that Ki67 index doesn't correctly reflect tumor grade.

\section{Treatment}

The treatment information was available for all 16 patients. Two patients refused surgical intervention owing to wide encroachment or metastasis; four patients were treated with radical tumor resection accompanied with pulmonary or right ventricular outlet tract reconstruction. Seven patients were treated with complete tumor resection, and 3 were treated with partial tumor resection owing to the invading into pulmonary artery medium or adventitia.

\section{Survival Analysis}

For all patients, the median follow-up duration was 11.5 months (range: 2 to 36 months). At multivariable analysis, MDM2 expression was the impact factor on PFS, but not on OS. EGFR, PDGFRA, age, tumor grade, proliferative index, and necrosis and surgery pattern were not the impact factors on PFS and OS. At univariate analysis, MDM2 expression had the negative association with PFS (correlation coefficient: $-0.546, p=0.029$ ). We also found that the tumors with MDM2 and EGFR coexpression had a shorter PFS compared with other tumors ( $3.13 \pm 2.30$ vs $15.25 \pm 12.80, \mathrm{p}=0.019)$. Also, MDMD and EGFR coexpression had a positive correlation with tumor metastasis (correlation coefficient: $0.5, \mathrm{p}=0.049$ ).

At the end of follow-up, 9 of 13 IS a patient died and 4 were alive. Of the 9 deaths, 1 patient died during the surgery procedure; 5 patients died of brain or lung metastasis after surgery; 2 patients without surgery died three months after diagnosis and one patient died suddenly without apparent death cause after 5 months. Four patients with recurrent tumor after surgery underwent radiotherapy and/or chemotherapy and were still alive at the end of follow-up. Of the three non-IS patients, 1 patient with grade 1 osteosarcoma patient was alive at the end of follow-up without recurrence and metastasis. The patient with grade I chondrosarcoma recurred 12 months after surgery and 1 patient with dedifferentiated chondrosarcoma patient underwent partial tumorectomy and died 3 months after surgery.

\section{Discussion}

In this study, we characterized the largest series of pulmonary artery sarcomas in China using a panel of immunohistochemical markers and genetic molecular study for a better classification.

In our study, we found that IS is the most common sarcoma arising in the pulmonary artery. IS has a higher tumor grade than other sarcomas. Most IS showed overexpression of MDM2, EGFR and PDGFRA. These features were also found in the previous studies [14-16]. On prognosis analysis, MDM2 had a negative prognostic significance which confirmed previous results in different case series [14-25]. In Pasello's study, the authors presented evidence that MDM2 can be an important diagnostic parameter to characterize a more aggressive phenotype of malignant mesothelioma [15]. In Lonardo, et al. study, MDM2 gene amplification was detected significantly more frequent in metastatic or recurrent osteosarcomas than it was in primary osteosarcomas [18]. Their data and our study suggested that MDM2 gene amplification may be associated with a worse progression and metastasis in sarcoma.

MDM2 amplification not only suggests that this pathway might play a relevant role in tumor pathogenesis, but also that MDM2 inhibition might be a potential treatment strategy in this disease. In a multi-institutional, international retrospective experience of systemic treatment in MDM2positive IS, tumors showed sensitivity to anthracyclinebased regimens, possibly greater than expected in other soft tissue sarcomas. PFS for advanced disease was 7.7 months, which was longer than IS patients in our study (3.3 months). Also, it is worth noting around $25 \%$ of patients with localized disease are expected to be alive and disease free at $>2$ years in this series. This suggests a possible role for neoadjuvant treatment in IS patients [19].

In the present study, the novel finding is that a positive correlation between EGFR expression and MDM2 expression. Also, MDM2 and EGFR coexpression was related to tumor metastasis and shorter PFS. The possible mechanism of this association is that MDM2 together with EGFR significantly decreased peroxisome proliferator-activated receptory (PPAR $\gamma$ ) protein levels [20]. PPAR $\gamma$ promotes cell growth arrest, differentiation and apoptosis in different types of cancer [21,22]. In addition, PPAR $\gamma$ suppresses cancer cell proliferation involved in inhibition of nuclear factor-kappa $\mathrm{B}$ (NF- $\mathrm{kB}$ ) or MUC1-C signaling [23-25]. In $\mathrm{Xu}$ and his colleagues' study, they found that EGFR-mediated PPAR $\gamma$-Y74 phosphorylation led to PPAR $\gamma$ ubiquitination and degradation by MDM2 ubiquitin ligase, which in turn increased binding of EGFR to NF- $\kappa \mathrm{B}$ and EGFR/NF- $\kappa \mathrm{B}$ signaling-mediated cell 


\section{Clinical Pathology \& Research Journal}

proliferation and survival [20]. PPAR $\gamma$ agonist pioglitazone can reverse this event [26]. Therefore, pioglitazone inhibited EGFR/MDM2-mediated cancer cell chemoresistance, which may provide a novel strategy for tumor treatment.

Primary pulmonary artery osteosarcoma and chondrosarcoma are very rare. Ysunezuka reviewed 8 cases of osteosarcoma in pulmonary artery including their own case in 2004 [27]. Clinically there is no gender predilection of primary pulmonary artery sarcoma, but 7 of 8 patients $(87.5 \%)$ of pulmonary artery osteosarcoma were women. In general, the prognosis of pulmonary osteosarcoma is poor and the recurrence rate is high. Of those 8 previously reported patients, 5 patients died within 1 year after admission. Four patients underwent surgery, but 2 patients died less than 1 year after surgery, 1 patient had recurrence 9 months postoperatively, and the outcome was not reported for 1 patient. Only 1 patient reported by Ysunezuka had no recurrence 24 months after pneumonectomy and resection of total right pulmonary artery [27]. Similar to that report, our patients who underwent radical surgery were alive 24 months after operation without recurrence. The prognostic feature of our case is similar to the low-grade central osteosarcoma in the skeleton [28].

Similarly, primary pulmonary artery chondrosarcoma is also very rare. There are approximal 6 cases reported till now [28-34]. Including our two cases, these 8 cases of primary pulmonary artery chondrosarcoma do not show sex predilection ( 4 females and 4 males). Also, the prognosis of primary pulmonary chondrosarcoma is poor and the recurrence and metastases rate are high. Of those 6 previously reported patients, all patients underwent tumor resection, 1 died shortly after surgery, 3 died within 2 years after surgery with recurrence or metastases, 1 was alive for 14 months with tumor free. One patient had recurrence within the same site of pulmonary trunk 2 years postoperatively. Similar to that report $[30,31]$, the patient with low grade chondrosarcoma in our group had recurrence 2 years after complete tumorectomy and recurrent mass was found within the same site of pulmonary artery. Once again this patient underwent tumor resection and discharged without symptom. Another case in our study, the patient with the histological features of dedifferentiated chondrosarcoma died 3 months postoperatively. The prognostic factor of pulmonary artery chondrosarcoma is the histologic grade which is also similar to the counterpart in the skeleton [35].

It is demonstrated that MDM2 amplification was found in low-grade osteosarcoma and some other types of osteosarcoma [18,34]. In the Lonardo i etal study, MDM2 gene amplification was detected significantly more frequently in metastatic or recurrent osteosarcomas than it was in primary osteosarcomas [18]. However, there is no report about the
MDM2 expression in pulmonary artery osteosarcoma due to its rarity. In our study, MDM2 expression was negative in the osteosarcoma. The further serial cases study need to be performed to discover the immunophenotype and genetic features of this rare sarcoma.

In conclusion, we find that is the most common type of pulmonary artery sarcoma. Immunologically, IS has a higher percentage of MDM2 and EGFR expression and coexpression of MDM2, EGFR and PDGFRA. MDM2 highly expressed and MDM2 and EGFR coexpression are the prognostic factors. Also, MDM2 and EGFR coexpression is significantly associated with tumor metastasis.

Limitations of this series include the retrospective nature of the analysis and small sample sizes of patients. Given the rarity of pulmonary artery sarcoma, and the small sample sizes, definitive conclusions are difficult to draw. The sample size is too small to find survival differences and a larger series, possibly with aggregated data from multiple institutions is necessary.

\section{Disclosure/Conflict of interest : There is NO conflict of interest to disclose.}

Funding: This research did not receive any specific grant from funding agencies in the public, commercial, or not-forprofit sectors.

\section{References}

1. Nonomura A, Kurumaya H, Kono N, Nakanuma Y, Ohta G, et al. (1988) Primary pulmonary artery sarcoma: report of two autopsy cases studied by immunohistochemistry and electron microscopy, and review of 110 cases reported in the literature. Acta Pathol Jpn 38(7): 883896.

2. Cox JE, Chiles C, Aquino SL, Savage P, Oaks T (1997) Pulmonary artery sarcomas: a review of clinical and radiologic features. J Comput Assist Tomogr 21(5): 750755.

3. Altman NH, Shelley WM (1973) Primary intimal sarcoma of the pulmonary artery. Johns Hopkins Med J. 133: 214222.

4. Burke AP, Virmani R (1992) Sarcomas of the great vessels. A clinicopathologic study. Cancer 71(5): 17611773.

5. Tavora F MM, Julie FSJ, Teri FJ, Allen BK (2008) Pulmonary Artery Sarcoma: a histologic and followup. The American Journal of Surgical Pathology 32: 1751-1761. 


\section{Clinical Pathology \& Research Journal}

6. Zhao JRJ, Bode Lesniewska B, Pfaltz M, Heitz PU, Komminoth P, et al. (2002) Combined comparative genomic hybridization and genomic microarray for detection of gene amplifications in pulmonary artery intimal sarcomas and adrenocortical tumors. Genes Chromosomes Cancer 34(1): 48-57.

7. Bode Lesniewska BZJ, Spee EJ, Biraima AM, Turina M, Komminoth P, et al. (2001) Gains of 12q13-14 and overexpression of $\mathrm{mdm} 2$ are frequent findings in intimal sarcomas of the pulmonary artery. Virchows Arch 438(1): 57-65.

8. Knuutila SBA, Autio K, Tarkkanen M, Wolf M, Monni O, et al. (1998) DNA copy number amplifications in human neoplasms review of comparative genomic hybridization studies. Am J Pathol 152(5): 1107-1123.

9. Tamborini E, Casieri P, Miselli F, Orsenigo M, Negri T, et al. (2007) Analysis of potential receptor tyrosine kinase targets in intimal and mural sarcomas. J Pathol 212(2): 227-235.

10. Doyle LA (2014) Sarcoma classification: an update based on the 2013 World Health Organization classification of tumors of soft tissue and bone. Cancer 120(12): 17631774.

11. Trojani MCG, Coindre JM, Coindre JM, Rouesse J, Bui NB, et al. (1984) Soft-tissue sarcomas of adults; study of pathological prognostic variables and definition of a histopathological grading system. Int J Cancer 33(1): 37 42.

12. Dewaele BFG, Finaletn Ferreiro J, Fletcher CD, Coindre JM, Guillou L, et al. (2010) Coactivated platelet-derived growth factor receptor \{alpha\} and epidermal growth factor receptor are potential therapeutic targets in intimal sarcoma. Cancer Res 70(18): 7304-7314.

13. Tajima S, Takanashi Y, Takahashi T, Neyatani H (2015) Intimal sarcoma of the abdominal aorta with plateletderived growth factor receptor alpha overexpression and amplification in mural invasive cells and pulmonary metastatic cells but not in intimal spreading cells. Pathol Int 65(8): 426-431.

14. Pasello G, Urso L, Mencoboni M, Grosso F, Ceresoli GL, et al. (2015) MDM2 and HIF1alpha expression levels in different histologic subtypes of malignant pleural mesothelioma: correlation with pathological and clinical data. Oncotarget 6(39): 42053-42066.

15. Nokitaka SMM, Susumu W, Fumihiko N, Eisuke K, Hirokazu C, et al. (2016) Primary Retroperitoneal Myxoid Liposarcomas. Am J Surg Pathol 40(9): 1286-1290.
16. Chen D, Zhu G, Wang D, Zhang Z, Fang W, et al. (2016) Clinicopathological and immunohistochemical features of pulmonary artery sarcoma: A report of three cases and review of the literature. Oncol Lett 11(4): 2820-2826.

17. Lonardo FUT, Huvos AG, Healey J, Ladanyi M (1997) P53 and MDM2 alterations in osteosarcomas: correlation with clinicopathologic features and proliferative rate. Cancer 79(8): 1541-1547.

18. Frezza AM, Assi T, Vullo SL, Ben Ami E, Dufresne A, et al. (2020) Systemic treatment in MDM2 positive intimal sarcoma: a multicentre experience with anthracycline, gemcitabine, and pazopanib within the World Sarcoma Network. Cancer 126(1): 98-104.

19. Ying X, Jianhua J, Wenbo Z, Zhi Z, Jiaming G, et al. (2016) EGFR/MDM2 signaling promotes NF-kB activation via PPAR $\gamma$ degradation. Carcinogenesis 37(2): 215-222.

20. Elstner E, Muller C, Koshizuka K, Williamson EA, Park D, et al. (1998) Ligands for peroxisome proliferatoractivated receptorgamma and retinoic acid receptor inhibit growth and induce apoptosis of human breast cancer cells in vitro and in BNX mice. Proc Natl Acad Sci USA 95(15): 8806-8811.

21. Sarraf P, Mueller E, Jones D, King FJ, DeAngelo DJ, et al. (1998) Differentiation and reversal of malignant changes in colon cancer through PPARgamma. Nat Med 4(9): 1046-1052.

22. Hou Y, Gao J, Xu H, Xu Y, Zhang Z, et al. (2014) PPARgamma E3 ubiquitin ligase regulates MUC1-C oncoprotein stability. Oncogene 33(49): 5619-5625.

23. Hou Y, Moreau F, Chadee $\mathrm{K}$ (2012) PPARgamma is an E3 ligase that induces the degradation of NFkappaB/p65. Nat Commun 3: 1300.

24. Xu H, You M, Shi H and Hou Y (2015) Ubiquitin-mediated NFkappaB degradation pathway. Cell Mol Immunol 12: 653-655.

25. Juanjuan S, Wenbo Z, Mengli $Y$, Ying $X$, Yongzhong $H$ (2016) Pioglitazone inhibits EGFR/MDM2 signalingmediated PPAR $\gamma$ degradation. Eur J Pharmacol 791: 316321.

26. Tsunezuka Y, Oda M, Takahashi M, Minato $\mathrm{H}$, Watanabe G (2004) Primary chondromatous osteosarcoma of the pulmonary artery. Ann Thorac Surg 77(1): 331-334.

27. Sakakibara K, Nakajima H, Kasai H (2015) A case of chondrosarcoma originating from the pulmonary artery. Asian Cardiovasc Thorac Ann 25(5): 398-399. 


\section{Clinical Pathology \& Research Journal}

28. Schleger $S$, Weingartner JS, Costi $M$, Eichinger WB (2012) A primary pulmonary artery chondrosarcoma manifesting as acute pulmonary embolism. Ann Thorac Surg 94(5): 1731-1733.

29. Wang F, Wang Y, Xue H, Zhang Y, Sun J, et al. (2014) Primary pulmonary artery chondrosarcoma: the use of different imaging modalities. Chin Med J (Engl) 127(15): 2868-2869.

30. Yamaguchi A, Ino T, Ide H, Mizuhara A, Kawahito, et al. (1993) Primary chondrosarcoma of the pulmonary artery. Nihon Kyobu Geka Gakkai Zasshi 41(2): 277-281.

31. Yamaguchi A, Ino T, Adachi H, Mizuhara A, Kawahito K et al. (1994) Reoperation for recurrent chondrosarcoma of the pulmonary trunk. Nihon Kyobu Geka Gakkai Zasshi 42(11): 2087-2090.

32. Hohbach C, Mall W (1977) Chondrosarcoma of the pulmonary artery. Beitr Pathol 160(3): 298-307.

33. Inwards C, Squire J (2014) Low-grade central osteosarcoma. In: Fletcher CDM, et al. (Eds), WHO classification of tumours of soft tissue and bone $4^{\text {th }}(\mathrm{edn})$, International Agency for Research on Cancer, France, pp: 281-282.

34. Hongendoorn PCW, Bovee JVMG, Nielsen GP (2014) Chondrosarcoma (grades I-III), including primary and secondary variants and periosteal chondrosarcoma. In: Fletcher CDM, et al. (Eds), WHO classification of tumours of soft tissue and bone, $4^{\text {th }}(\mathrm{edn})$, International Agency for Research on Cancer, France, pp: 264-267.

35. Inwards C, Hogendoorn PCW (2014) Differentiated chondrosarcoma. In: Fletcher CDM, et al. WHO classification of tumours of soft tissue and bone, $4^{\text {th }}(\mathrm{edn})$, International Agency for Research on Cancer, France, pp: 269-270.

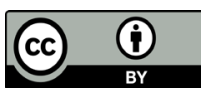

